\title{
Nutrients Behavior From the Association Pig Slurry and Chemical Fertilizers on Soybean Crop
}

\author{
Adriana Smanhotto ${ }^{1 *}$, Silvio César Sampaio ${ }^{2}$, Tatiane Cristina Dal Bosco ${ }^{3}$, Maritane Prior ${ }^{4}$ \\ and Rosimaldo Soncela ${ }^{1}$ \\ ${ }^{I}$ Eixo de Recursos Naturais; Instituto Federal de Mato Grosso do Sul; Nova Andradina - MS - Brasil. ${ }^{2}$ Programa de \\ Pós Graduação em Engenharia Agrícola; Universidade Estadual do Oeste do Paraná; Cascavel - PR - Brasil. \\ ${ }^{3}$ Universidade Tecnológica Federal do Paraná; Londrina - PR - Brasil. ${ }^{4}$ Centro de Ciências Agrárias; \\ Universidade Estadual do Oeste do Paraná; Marechal Cândido Rondon - PR - Brasil
}

\begin{abstract}
This study aimed at evaluating the effects of pig slurry application with mineral fertilizer on ions leaching from the soil in soybean crop. The experiment was carried out in 24 drainage lysimeters under protection. The soybean cultivar CD 202/COODETEC was sown in a soil that received 0, 100, 200 and $300 \mathrm{~m}^{3} \mathrm{ha}^{-1}$ of pig slurry in one cycle, with or without mineral fertilizer. There were three samplings of soil throughout the trial to determine the $\mathrm{pH}$, $\mathrm{N}, \mathrm{NO}_{3}^{-}, \mathrm{K}^{+}, \mathrm{Ca}^{2+}, \mathrm{Mg}^{2+}, \mathrm{Na}^{+}, \mathrm{Cu}^{+2}, \mathrm{Zn}^{+2}, \mathrm{OM}, \mathrm{CEC}, \mathrm{EC}$ and SAR six times during the crop cycle. The yield was determined in the plants. In soil, pig slurry increased the concentrations of $\mathrm{pH}, \mathrm{NO}_{3}^{-}, \mathrm{K}^{+}, \mathrm{Zn}^{+2}, \mathrm{OM}$ and CEC, while mineral fertilizer increased $P$ and $\mathrm{Zn}^{+2}$ concentrations. The limits observed for the leachate parameters did not present environmental problems according to the Brazilian legislation, but in the intermediate and long term, there special attention should be given to $\mathrm{NO}_{3}^{-}, \mathrm{P}, \mathrm{Na}^{+}, \mathrm{EC}$ and $\mathrm{SAR}$. Soybean yield was higher with mineral fertilizer and increased with pig slurry application.
\end{abstract}

Key words: organic fertilization, soil pollution, environment contamination, soybean culture

\section{INTRODUCTION}

Swine sector is an alternative of extra income for small farmers with familiar labor (Gatiboni et al. 2008). Its wastewaters need special attention due to the volume generated (Deng et al. 2007) in the farms as well as its organic load (Oliveira et al. 2000). Among the available technologies to treat the wastewater, there is the wastewater disposing on soil, which has been used on a large scale. This method uses the soil-plant system for the degradation, assimilation and immobilization of wastewater constituents (Medeiros et al. 2005). Pig slurry is used as fertilizer in the areas of grain and pasture annual crops. This is a benefic situation, because the nutrients from the wastewater are reused in the same production area (Müller et al. 2007). However, in many farms, the produced waste amount exceeds soil carrying capacity and changes from the fertilizer to environmental pollutant (Mattias et al. 2010).

There are several studies on the environmental impact of pig slurry application, since it can cause ions accumulation on soil profile, fertility problems (Sampaio et al. 2010a; Caovilla et al. 2010), as well as increase the concentration in the surface and groundwater due to leaching (Anami et al. 2008; Prior et al. 2009; Smanhotto et al. 2010; Sampaio et al. 2010b; Maggi et al. 2011) and runoff (Gomes et al. 2004; Caovilla et al.

*Author for correspondence: adriana.smanhotto@ifms.edu.br 
2005; Dal Bosco et al. 2008; Doblinski et al. 2010). Among the elements, nitrogen and its forms can stay and get concentrated in the soil, thus it could pose a potential risk. Usually, wastewater has high levels of nitrogen, which induce a continuous monitoring of its mineralization process in the soil (Daudén and Quílez 2004; Bergström and Kirchmann 2006; Askegaard et al. 2011). Other elements also need special attention such as salinity and sodicity (Díez et al. 2004; Freitas et al. 2005), phosphorus (Hountin et al. 2000; Heathwaite et al. 2000; Djodjic et al. 2004; Basso et al. 2005; Bergström and Kirchmann 2006; Ceretta et al. 2010), potassium, calcium, magnesium, sodium, (Ribeiro and Galbiatti 2004; Queiroz et al. 2004), copper and zinc (Hsu and Lo 2000; Jondreville et al. 2003; Graber et al. 2005; Ashworth and Alloway 2007; Mattias et al. 2010). On the other hand, other studies have shown the benefits of applying pig slurry in soil-plant system (Scherer et al. 2007; Assmann et al. 2007; Hao et al. 2008). In Brazil, however, the trials are limited to corn crops (Daudén and Quílez 2004; Giacomini et al. 2009) and pastures (Buckey et al. 2010). According to Wohlenberg et al. (2004), the crop rotation with pasture and leguminous promotes better conservation of the soil. This is due to grass root system and decomposition rate of leguminous; thus, if soybean is taken as an example, its decomposition rate is enhanced due to a symbiosis with bacteria of Bradyrhizobium genus (Vieira et al. 2005).

Considering that the western Paraná has an intense yield of soybean and corn combined with pig slurry application on the soil, this study aimed at evaluating its effects on the soil and ions leaching since it is mostly applied with mineral fertilizer in soybean crop.

\section{MATERIAL AND METHODS}

The experiment was carried out at the Experimental Center of Agricultural Engineering NEEA, Western Paraná State University UNIOESTE. The geographic coordinates are: $24^{\circ}$ $54^{\prime}$ southern latitude, $53^{\circ} 32^{\prime}$ western longitude and altitude of 760 meters. According to Köeppen classification, the studied region has a super humid mesothermal subtropical weather, whose annual average of rainfall is $1800 \mathrm{~mm}$, with hot summers, rare frosts and rainfalls occur mainly in summertime; however, there is no specific dry season. The average temperature is $20^{\circ} \mathrm{C}$ and relative humidity ranges in $75 \%$ (IAPAR, 2000). Its soil was classified as typical Rhodic Hapludox (EMBRAPA, 2006). The soybean cultivar CD 202/COODETEC was sown in a 118 day early cycle. Sowing was manual and there were 15 seeds per meter (density). The mineral fertilizer (MF) at sowing was $250 \mathrm{~kg} \mathrm{ha}^{-1}$ according to $0-20-20$ formula and soil analyses. All the soil analyses followed the protocol of Raij et al. (2001). The experiment was carried out in 24 drainage lysimeters under a shelter. Each drainage lysimeter was taken as an experimental plot, with $1 \mathrm{~m}^{3}$ volume and $1.60 \mathrm{~m}^{2}$. The plants were irrigated by drip system and the side lines were laid along the crop rows. Irrigation was performed at $14,44,58$, $72,86,100$ and 114 days after the sowing (DAS). The amount of applied water through the irrigation simulated an average rainfall from the region, resulting in water depths of 72, 79, 112, 91, 81, 65 and $76 \mathrm{~mm}$ (Longo et al. 2006). The applied pig slurry (PS) (Table 1) underwent treatment in a biodigester, followed by a sedimentation tank and a stabilization pond.

The treatments applied to the plots consisted of four doses of PS application (0, 100, 200, 300 $\mathrm{m}^{3} \mathrm{ha}^{-1}$ cycle) and two levels of MF (0 and $100 \%$ of mineral fertilizer at seeding). The PS doses were separated into six applications throughout the crop cycle.

Soil samples were collected from each lysimeter at 0-60 cm depth, in order to cover the whole profile of the experimental plot. Samples were collected at 0 DAS (before soybean sowing), at 59 DAS and at 118 DAS (at the end of soybean cycle) and were analyzed for the organic matter $(\mathrm{OM})$, cation exchange capacity (CEC), $\mathrm{pH}, \mathrm{N}, \mathrm{NO}_{3}^{-}, \mathrm{K}^{+}, \mathrm{Ca}^{2+}$, $\mathrm{Mg}^{2+}, \mathrm{Na}+, \mathrm{Cu}^{+2}, \mathrm{Zn}^{+2}, \mathrm{EC}$ and SAR were determined. In each plot, samples were collected from the leachate sis times at $14,44,58,72,86$, 100 and 114 DAS, always after the irrigation. Based on the leachate material, $\mathrm{pH}, \mathrm{N}, \mathrm{NO}_{3}^{-}, \mathrm{K}^{+}$, $\mathrm{Ca}^{2+}, \mathrm{Mg}^{2+}, \mathrm{Na}+, \mathrm{Cu}^{+2}, \mathrm{Zn}^{+2}, \mathrm{EC}$ and SAR were also determined. For the soybean crop, the yield was evaluated from all the seeds that were obtained in the experimental plot at 118 DAS. The grain weight was adjusted to $13 \%$ humidity.

Data descriptive analysis and control of error normality by Anderson-Darling test were obtained before the analysis of variance. Data transformations were done for the parameters that did not show normal distribution. The experimental design was in randomized blocks, 
factorial design (4x2), four levels of PS and two levels of MF, with three replications. Tukey test, at $5 \%$ probability, was used to obtain the means. The aforementioned experimental design and mean test were singly applied in each sampling for the soil, leachate and plant.

Table 1 - Selected properties of pig slurry.

\begin{tabular}{|c|c|}
\hline Parameters & Value \\
\hline $\mathrm{pH}_{(\mathrm{CaCl} 2)}$ & 7.73 \\
\hline $\mathrm{N}\left(\mathrm{mg} . \mathrm{L}^{-1}\right)$ & 801.67 \\
\hline $\mathrm{NH}_{3}\left(\mathrm{mg} \cdot \mathrm{L}^{-1}\right)$ & 556.70 \\
\hline $\mathrm{NO}_{3}^{-}\left(\mathrm{mg} \cdot \mathrm{L}^{-1}\right)$ & 1.52 \\
\hline $\mathrm{NO}_{2}^{-}\left(\mathrm{mg} \cdot \mathrm{L}^{-1}\right)$ & 2.03 \\
\hline $\mathrm{P}\left(\mathrm{mg} \cdot \mathrm{L}^{-1}\right)$ & 92.19 \\
\hline $\mathrm{K}^{+}\left(\mathrm{mg} \cdot \mathrm{L}^{-1}\right)$ & 543.33 \\
\hline $\mathrm{Na}^{+}\left(\mathrm{mg} \cdot \mathrm{L}^{-1}\right)$ & 18.20 \\
\hline $\mathrm{Ca}^{2+}\left(\mathrm{mg} \cdot \mathrm{L}^{-1}\right)$ & 50.97 \\
\hline $\mathrm{Mg}^{2+}\left(\mathrm{mg} \cdot \mathrm{L}^{-1}\right)$ & 23.77 \\
\hline $\mathrm{Cu}^{+2}\left(\mathrm{mg} . \mathrm{L}^{-1}\right)$ & 0.20 \\
\hline $\mathrm{Zn}^{+2}\left(\mathrm{mg} \cdot \mathrm{L}^{-1}\right)$ & 1.17 \\
\hline $\mathrm{EC}\left(\mathrm{dS} \cdot \mathrm{m}^{-1}\right)$ & 4.89 \\
\hline SAR (meq. $L^{-1}$ ) & 2.98 \\
\hline BOD (mg. $\left.\mathrm{L}^{-1}\right)$ & 671.04 \\
\hline COD (mg. $\left.\mathrm{L}^{-1}\right)$ & $1,444.07$ \\
\hline $\mathrm{TS}\left(\mathrm{mg} \cdot \mathrm{L}^{-1}\right)$ & 2640.00 \\
\hline TFS (mg.L $\left.\mathrm{L}^{-1}\right)$ & $1,346.67$ \\
\hline TVS (mg.L $\left.\mathrm{L}^{-1}\right)$ & $1,293.33$ \\
\hline TDS (mg.L $\left.{ }^{-1}\right)$ & $1,885.67$ \\
\hline
\end{tabular}

APHA, AWWA \& WEF Protocol (1998); $\mathrm{N}$ : Nitrogen, $\mathrm{NH}_{3}$ : ammonia nitrogen; $\mathrm{NO}_{3}{ }^{-}$: nitrate; $\mathrm{NO}_{2}{ }^{-}$: Nitrite; P: Phosphorus; $\mathrm{K}^{+}$: Potassium; $\mathrm{Na}^{+}$: Sodium; $\mathrm{Ca}^{2+}$ : Calcium; $\mathrm{Mg}^{2+}$ : Magnesium; $\mathrm{Cu}^{+2}$ : Copper; $\mathrm{Zn}^{+2}$ : Zinc; EC: electrical conductivity; SAR: sodium adsorption ratio; BOD: biochemical Oxygen Demand; COD: chemical oxygen demand; TS: total solids; TFS: total fixed solids; TVS: total volatile solids; TDS: total dissolved solids.

\section{RESULTS AND DISCUSSION}

\section{Soil parameters}

Nitrogen did not show a significant variation when compared with the treatments and sampling periods during the soybean development (Table 2). However, $\mathrm{N}$ concentrations increased with $\mathrm{MF}$ and PS, but decreased at the end of the cycle (Table 2). This unstable behavior in the soil could be related to $\mathrm{N}$ changes in $\mathrm{N}$ form such as losses by ammonia volatilization, nitrate leaching, denitrification and runoff (Aita et al. 2004; Sampaio et al. 2010a). Another important factor in such behavior is $\mathrm{N}$ ratio as ammonia, which is 40 to $70 \%$ (Scherer et al. 1996). Thus, $\mathrm{N}$ dynamics in the soil is hard to be predicted in a short term (Raij 1991).

The $\mathrm{NO}_{3}{ }^{-}$at 59 and 118 DAS (Table 2) showed a significant change, which, decreased over time, except for both the treatments with 0 PS, which increased $\mathrm{NO}_{3}{ }^{-}$concentration after crop sampling. This was probably due to the mineralization process of $\mathrm{N}$ on the soil, and hence $\mathrm{NO}_{3}$ generation, which allowed its slight leaching
(Table 3). Primavesi et al. (2006) reported that $\mathrm{NO}_{3}{ }^{-}$in the soil was not absorbed by the plants nor immobilized by the soil microbes, so it could be easily leached due to its negative charge as well as non-adsorption by the soil colloids that had predominantly negative charges.

Rossi et al. (2007) reported that $\mathrm{NO}_{3}{ }^{-}$mobility was mainly dependent on the mass flow and probably this had happened during the present study. Since at 0 DAS (Table 2), its concentration was higher than after sowing at 59 DAS and 118 DAS when throughout the experiment, the water depths were $72,79,112,91,81,65$ and $76 \mathrm{~mm}$. So, $\mathrm{NO}_{3}{ }^{-}$ movement in the soil was also influenced by the factors that changed water in the soil such as porosity and structure.

The $\mathrm{pH}$ of aqueous extract on the soil did not change with MF, but changed with the PS doses at 59 and 118 DAS (Table 2). Soil sample at 0 DAS (Table 2) showed no significant differences. Guarçoni and Mendonça (2003), Caires et al. (2004) and Assmann et al. (2007) $(\mathrm{N}=2100$ mg. $\left.\mathrm{L}^{-1}\right)$ also observed an increase in $\mathrm{pH}$ with PS 
application on the soil. According to Raij (1991), all the means were classified as very low acidity, or $\mathrm{pH} 6.1$.

At 59 and 118 DAS (Table 2), the pH increased with OM increase. This was due to the presence of organic compounds that significantly increase the availability of carbon in the soil (Hue and Licudine 1999). Asmann et al. (2007) $(\mathrm{N}=2100$ mg. $\mathrm{L}^{-1}$ ) observed no increase in OM content with
PS application. According to these authors, the intrinsic characteristics of the studied waste should be considered because the quality of organic compounds could determine the OM concentration in the soil. These organic compounds are easily mineralized as they get oxidized in a few days, or weeks. This happens because there is an increase in the microbial activity from the applied waste.

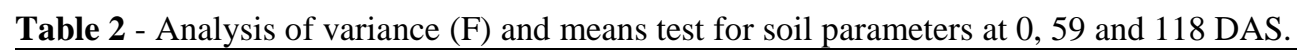

\begin{tabular}{|c|c|c|c|c|c|c|c|}
\hline \multicolumn{8}{|c|}{ 0 DAS } \\
\hline & $\begin{array}{c}\mathbf{p H} \\
\left(\mathrm{CaCl}_{2}\right)\end{array}$ & $\begin{array}{c}\mathbf{N} \\
\left(\mathrm{mg} . \mathrm{L}^{-1}\right)\end{array}$ & $\begin{array}{c}\mathrm{NO}_{3}^{-} \\
(\mathrm{mg.L}\end{array}$ & $\begin{array}{c}\mathbf{K}^{+} \\
\left(\mathrm{mg.dm}^{-3}\right)\end{array}$ & $\begin{array}{c}\dagger \mathrm{Ca}^{+2} \\
(\mathrm{mg.dm}\end{array}$ & $\begin{array}{c}\dagger \mathrm{Mg}^{+2} \\
\left(\mathrm{mg}^{-\mathbf{d m}^{-3}}\right)\end{array}$ & $\begin{array}{c}\dagger \mathrm{Na}^{+} \\
\left(\mathrm{mg.dm}^{-3}\right)\end{array}$ \\
\hline$\S 0$ & $6.45 \mathrm{a}$ & $1868.84 a$ & $46.82 \mathrm{a}$ & $85.97 \mathrm{a}$ & $147.77 \mathrm{a}$ & $94.93 \mathrm{a}$ & $3.53 \mathrm{a}$ \\
\hline$\S 100$ & $6.58 \mathrm{a}$ & $1884.40 \mathrm{a}$ & $46.90 \mathrm{a}$ & $94.86 \mathrm{ab}$ & $80.50 \mathrm{a}$ & $58.03 \mathrm{a}$ & $3.18 \mathrm{a}$ \\
\hline$\$ 200$ & $6.58 \mathrm{a}$ & $1755.28 \mathrm{a}$ & $48.98 \mathrm{a}$ & $144.26 \mathrm{bc}$ & $99.57 \mathrm{a}$ & $73.61 \mathrm{a}$ & $3.48 \mathrm{a}$ \\
\hline$\$ 300$ & $6.67 \mathrm{a}$ & $1824.67 \mathrm{a}$ & $51.87 \mathrm{a}$ & $151.70 \mathrm{c}$ & $110.18 \mathrm{a}$ & $72.98 \mathrm{a}$ & $4.23 \mathrm{a}$ \\
\hline$\S \mathrm{A}$ & $6.55 \mathrm{~A}$ & $1815.49 \mathrm{~A}$ & $48.91 \mathrm{a}$ & $118.09 \mathrm{~A}$ & $64.33 \mathrm{~A}$ & $71.14 \mathrm{~A}$ & $3.41 \mathrm{~A}$ \\
\hline$\S \mathrm{P}$ & $6.59 \mathrm{~A}$ & $1851.11 \mathrm{~A}$ & $48.37 \mathrm{a}$ & $120.31 \mathrm{~A}$ & $76.25 \mathrm{~A}$ & $78.64 \mathrm{~A}$ & $3.80 \mathrm{~A}$ \\
\hline PS & $2.33^{\mathrm{ns}}$ & $0.45^{\mathrm{ns}}$ & $1.16^{\mathrm{ns}}$ & $7.81^{*}$ & $3.05^{\mathrm{ns}}$ & $2.01^{\mathrm{ns}}$ & $0.67^{\mathrm{ns}}$ \\
\hline MF & $0.50^{\mathrm{ns}}$ & $0.17^{\mathrm{ns}}$ & $0.06^{\mathrm{ns}}$ & $0.03^{\mathrm{ns}}$ & $0.05^{\mathrm{ns}}$ & $0.28^{\mathrm{ns}}$ & $0.62^{\mathrm{ns}}$ \\
\hline PS x MF & $2.82^{\mathrm{ns}}$ & $1.25^{\mathrm{ns}}$ & $0.56^{\mathrm{ns}}$ & $0.68^{\mathrm{ns}}$ & $2.81^{\mathrm{ns}}$ & $2.95^{\mathrm{ns}}$ & $0.30^{\mathrm{ns}}$ \\
\hline Block & $0.26^{\mathrm{ns}}$ & $0.42^{\mathrm{ns}}$ & $6.85^{*}$ & $1.96^{\mathrm{ns}}$ & $3.31^{\mathrm{ns}}$ & $2.44^{\mathrm{ns}}$ & $0.49^{\mathrm{ns}}$ \\
\hline $\mathrm{CV}$ & 2.19 & 11.49 & 11.08 & 24.69 & 17.23 & 16.45 & 16.85 \\
\hline \multicolumn{8}{|c|}{$59 \mathrm{DAS}$} \\
\hline$\S 0$ & $6.18 \mathrm{a}$ & $1919.87 \mathrm{a}$ & $14.05 \mathrm{a}$ & $75.60 \mathrm{a}$ & $74.83 a$ & $45.00 \mathrm{a}$ & $2.78 \mathrm{a}$ \\
\hline$\S 100$ & $6.23 \mathrm{~b}$ & $2144.70 \mathrm{a}$ & $25.13 \mathrm{~b}$ & $117.30 \mathrm{~b}$ & $69.16 \mathrm{a}$ & $39.83 \mathrm{a}$ & $3.11 \mathrm{a}$ \\
\hline$\$ 200$ & $6.57 \mathrm{~b}$ & $2056.93 a$ & $31.79 \mathrm{bc}$ & $125.77 \mathrm{~b}$ & $73.83 a$ & $42.83 a$ & $2.60 \mathrm{a}$ \\
\hline$\$ 300$ & $6.67 \mathrm{~b}$ & $2360.87 \mathrm{a}$ & $42.52 \mathrm{c}$ & $117.95 \mathrm{~b}$ & $63.33 \mathrm{a}$ & $37.83 \mathrm{a}$ & $2.65 \mathrm{a}$ \\
\hline$\S \mathrm{A}$ & $6.35 \mathrm{~A}$ & $2204.72 \mathrm{~A}$ & $28.14 \mathrm{~A}$ & 86.68 A & $64.33 \mathrm{~A}$ & $37.83 \mathrm{~A}$ & $2.87 \mathrm{~A}$ \\
\hline$\S \mathrm{P}$ & $6.47 \mathrm{~A}$ & $2036.47 \mathrm{~A}$ & $28.60 \mathrm{~A}$ & 131.63B & $76.25 \mathrm{~A}$ & $44.92 \mathrm{~A}$ & $2.70 \mathrm{~A}$ \\
\hline- & - & - & - & - & - & - & - \\
\hline- & - & - & - & - & - & - & - \\
\hline PS & $9.94^{*}$ & $1.18^{\mathrm{ns}}$ & $19.85^{*}$ & $7.09^{*}$ & $0.14^{\mathrm{ns}}$ & $0.12^{\mathrm{ns}}$ & $0.99^{\mathrm{ns}}$ \\
\hline $\mathrm{MF}$ & $2.69^{\mathrm{ns}}$ & $0.98^{\text {ns }}$ & $0.03^{\mathrm{ns}}$ & $27.80^{*}$ & $0.90^{\mathrm{ns}}$ & $0.48^{\mathrm{ns}}$ & $0.56^{\mathrm{ns}}$ \\
\hline $\mathrm{PS} \times \mathrm{MF}$ & $1.09^{\mathrm{ns}}$ & $0.99^{\text {ns }}$ & $0.23^{\mathrm{ns}}$ & $2.60^{\mathrm{ns}}$ & $1.01^{\mathrm{ns}}$ & $0.65^{\mathrm{ns}}$ & $0.78^{\mathrm{ns}}$ \\
\hline Block & $0.47^{\mathrm{ns}}$ & $9.29^{*}$ & $0.05^{\mathrm{ns}}$ & $1.54^{\mathrm{ns}}$ & $0.59^{\mathrm{ns}}$ & $0.37^{\mathrm{ns}}$ & $1.54^{\mathrm{ns}}$ \\
\hline $\mathrm{CV}(\%)$ & 2.91 & 19.64 & 23.13 & 19.14 & 20.90 & 25.31 & 9.90 \\
\hline \multicolumn{8}{|c|}{$118 \mathrm{DAS}$} \\
\hline$\S 0$ & $6.22 \mathrm{a}$ & 1996.40a & $17.94 \mathrm{a}$ & $65.17 \mathrm{a}$ & $62.83 a$ & $35.16 a$ & $2.65 a$ \\
\hline$\S 100$ & $6.58 \mathrm{~b}$ & $1993.13 \mathrm{a}$ & $23.40 \mathrm{ab}$ & $80.80 \mathrm{a}$ & $81.67 \mathrm{a}$ & $44.50 \mathrm{a}$ & $2.47 \mathrm{a}$ \\
\hline$\$ 200$ & $6.72 \mathrm{~b}$ & $2091.60 \mathrm{a}$ & $27.26 \mathrm{ab}$ & $76.25 \mathrm{a}$ & $64.83 a$ & $35.50 \mathrm{a}$ & $2.82 \mathrm{a}$ \\
\hline$\$ 300$ & $6.78 \mathrm{~b}$ & $2142.00 \mathrm{a}$ & $30.09 \mathrm{~b}$ & $108.17 \mathrm{~b}$ & $82.83 a$ & $50.67 \mathrm{a}$ & $2.42 \mathrm{a}$ \\
\hline$\S \mathrm{A}$ & $6.57 \mathrm{~A}$ & $1995.70 \mathrm{~A}$ & $23.95 \mathrm{~A}$ & $64.52 \mathrm{~A}$ & $80.42 \mathrm{~A}$ & $45.50 \mathrm{~A}$ & $2.85 \mathrm{~A}$ \\
\hline$\S \mathrm{P}$ & $6.57 \mathrm{~A}$ & $2115.87 \mathrm{~A}$ & $25.39 \mathrm{~A}$ & $100.68 B$ & $65.67 \mathrm{~A}$ & $37.42 \mathrm{~A}$ & $2.33 \mathrm{~A}$ \\
\hline- & - & - & - & - & - & - & - \\
\hline- & - & - & - & - & - & - & - \\
\hline PS & $11.10^{*}$ & $0.36^{\mathrm{ns}}$ & $3.95^{*}$ & $19.09^{*}$ & $0.56^{\mathrm{ns}}$ & $0.64^{\mathrm{ns}}$ & $0.99^{\mathrm{ns}}$ \\
\hline MF & $0.01^{\mathrm{ns}}$ & $0.96^{\mathrm{ns}}$ & $0.29^{\mathrm{ns}}$ & $74.79^{*}$ & $0.98^{\mathrm{ns}}$ & $0.65^{\mathrm{ns}}$ & $0.56^{\mathrm{ns}}$ \\
\hline PS x MF & $0.06^{\mathrm{ns}}$ & $0.32^{\mathrm{ns}}$ & $0.56^{\mathrm{ns}}$ & $1.18^{\mathrm{ns}}$ & $1.92^{\mathrm{ns}}$ & $0.93^{\mathrm{ns}}$ & $0.78^{\mathrm{ns}}$ \\
\hline Block & $1.77^{\mathrm{ns}}$ & $0.23^{\mathrm{ns}}$ & $16.54^{*}$ & $6.13^{*}$ & $0.12^{\mathrm{ns}}$ & $0.97^{\mathrm{ns}}$ & $1.54^{\mathrm{ns}}$ \\
\hline $\mathrm{CV}(\%)$ & 2.83 & 14.58 & 26.27 & 12.40 & 21.41 & 25.78 & 9.90 \\
\hline
\end{tabular}

$\S$ : means $(n=6)$ followed by the same lower-case letters in column do not differ by Tukey test at $5 \%$ significance for PS and means $(n=$ 12) followed by the same upper-case letters in column do not differ by Tukey test at $5 \%$ significance for MF.

I: means $(n=3)$ followed by the same lower-case letters in column do not differ by Tukey test at $5 \%$ significance for interaction sliced PS in MF and the same upper-case letters in column do not differ in Tukey test at 5\% significance for interaction sliced MF in PS.

$\dagger$ : Transformation $x^{1 / 2}, \mathrm{~A}$ : absence of MF, P: presence of MF *: Significant at 5\%; ns: not significant at 5\%, CV: coefficient of variation. 
$\underline{\text { Table } 3 \text { - Analysis of variance }(\mathrm{F}) \text { and means test for soil parameters at } 0,59 \text { and } 118 \text { DAS. }}$

\begin{tabular}{|c|c|c|c|c|c|c|c|c|}
\hline \multicolumn{9}{|c|}{0 DAS } \\
\hline & $\begin{array}{c}\dagger \mathrm{Cu}^{+2} \\
\left(\mathrm{mg} \cdot \mathrm{dm}^{-3}\right)\end{array}$ & $\begin{array}{c}\mathrm{Zn}^{+2} \\
\left(\mathrm{mg} \cdot \mathrm{dm}^{-3}\right)\end{array}$ & $\begin{array}{c}\mathrm{OM} \\
\left(\mathrm{g} \cdot \mathrm{dm}^{-3}\right)\end{array}$ & $\begin{array}{c}\text { CEC } \\
\left(\mathrm{mmol}^{\left.-\mathrm{dm}^{-1}\right)}\right. \\
\end{array}$ & $\begin{array}{c}\dagger \mathrm{EC} \\
\left(\mathrm{dS} . \mathrm{m}^{-1}\right)\end{array}$ & $\begin{array}{c}\dagger \text { SAR } \\
\left(\mathrm{meq} \cdot \mathrm{L}^{-1}\right)\end{array}$ & $\begin{array}{l}- \\
- \\
\end{array}$ & $\begin{array}{c}\dagger \mathrm{P} \\
\left(\mathrm{mg} \cdot \mathrm{dm}^{-3}\right)\end{array}$ \\
\hline$\$ 0$ & $5.05 a$ & $0.50 \mathrm{a}$ & $23.33 a$ & $129 a$ & $0.11 \mathrm{a}$ & $0.34 \mathrm{a}$ & - & $16.17 \mathrm{a}$ \\
\hline$\S 100$ & $5.19 \mathrm{a}$ & $0.43 a$ & $23.17 \mathrm{a}$ & $137 \mathrm{a}$ & $0.10 \mathrm{a}$ & $0.39 a$ & - & $22.66 a$ \\
\hline$\S 200$ & $5.19 \mathrm{a}$ & $0.53 a$ & $23.17 \mathrm{a}$ & $143 a$ & $0.08 \mathrm{a}$ & $0.38 \mathrm{a}$ & - & $17.00 \mathrm{a}$ \\
\hline$\S 300$ & $5.20 \mathrm{a}$ & $0.55 a$ & $24.67 \mathrm{a}$ & $147 \mathrm{a}$ & $0.11 \mathrm{a}$ & $0.48 \mathrm{a}$ & - & $16.66 \mathrm{a}$ \\
\hline$\S \mathrm{A}$ & $5.49 \mathrm{~A}$ & $0.54 \mathrm{~A}$ & $24.42 \mathrm{~A}$ & $140 \mathrm{~A}$ & $0.11 \mathrm{~A}$ & $0.38 \mathrm{~A}$ & - & $19.91 \mathrm{~A}$ \\
\hline$\S \mathrm{P}$ & $4.83 \mathrm{~A}$ & $0.47 \mathrm{~A}$ & $22.75 \mathrm{~A}$ & $138 \mathrm{~A}$ & $0.10 \mathrm{~A}$ & $0.42 \mathrm{~A}$ & - & $16.33 \mathrm{~A}$ \\
\hline PS & $0.03^{\mathrm{ns}}$ & $0.78^{\mathrm{ns}}$ & $0.48^{\mathrm{ns}}$ & $2.07^{\mathrm{ns}}$ & $0.96^{\mathrm{ns}}$ & $0.53^{\mathrm{ns}}$ & - & $0.65^{\mathrm{ns}}$ \\
\hline MF & $0.68^{\mathrm{ns}}$ & $0.81^{\mathrm{ns}}$ & $2.50^{\mathrm{ns}}$ & $0.11^{\mathrm{ns}}$ & $0.49^{\mathrm{ns}}$ & $0.34^{\mathrm{ns}}$ & - & $1.24^{\mathrm{ns}}$ \\
\hline $\mathrm{PS} \times \mathrm{MF}$ & $2.23^{\mathrm{ns}}$ & $2.52^{\mathrm{ns}}$ & $0.98^{\mathrm{ns}}$ & $1.27^{\mathrm{ns}}$ & $0.14^{\mathrm{ns}}$ & $0.46^{\mathrm{ns}}$ & - & $0.42^{\mathrm{ns}}$ \\
\hline Block & $5.96^{\mathrm{ns}}$ & $2.32^{\mathrm{ns}}$ & $6.79^{\mathrm{ns}}$ & $0.59^{\mathrm{ns}}$ & $3.58 *$ & $0.42^{\mathrm{ns}}$ & - & $3.39^{*}$ \\
\hline $\mathrm{CV}$ & 18.08 & 18.78 & 10.95 & 9.30 & 15.09 & 21.02 & - & 22.14 \\
\hline \multicolumn{9}{|c|}{59 DAS } \\
\hline$\$ 0$ & $4.48 \mathrm{a}$ & $0.60 \mathrm{a}$ & $25.50 \mathrm{a}$ & $122 \mathrm{a}$ & $0.09 a$ & $0.39 a$ & I0 A & $15.67 \mathrm{aA}$ \\
\hline$\$ 100$ & $4.60 \mathrm{a}$ & $1.10 \mathrm{a}$ & $27.00 \mathrm{a}$ & $144 \mathrm{ab}$ & $0.14 \mathrm{a}$ & $0.42 \mathrm{a}$ & I0 P & $37.67 \mathrm{aB}$ \\
\hline$\S 200$ & $4.60 \mathrm{a}$ & $1.17 \mathrm{a}$ & $28.50 \mathrm{ab}$ & $153 a b$ & $0.13 \mathrm{a}$ & $0.38 \mathrm{a}$ & II100 A & $28.33 \mathrm{abA}$ \\
\hline$\$ 300$ & $4.88 \mathrm{a}$ & $2.70 \mathrm{~b}$ & $30.83 \mathrm{~b}$ & $171 \mathrm{~b}$ & $0.18 \mathrm{a}$ & $0.38 \mathrm{a}$ & II100 P & $45.33 \mathrm{abB}$ \\
\hline$\S \mathrm{A}$ & $4.89 \mathrm{~A}$ & $1.47 \mathrm{~A}$ & $27.42 \mathrm{~A}$ & $152 \mathrm{~A}$ & $0.12 \mathrm{~A}$ & $0.42 \mathrm{~A}$ & $\mathbb{I}[200 \mathrm{~A}$ & $35.67 \mathrm{bA}$ \\
\hline$\S \mathrm{P}$ & $4.39 \mathrm{~A}$ & $1.47 \mathrm{~A}$ & $28.50 \mathrm{~A}$ & $156 \mathrm{~A}$ & $0.15 \mathrm{~A}$ & $0.37 \mathrm{~A}$ & I[200 P & $47.00 \mathrm{abB}$ \\
\hline- & - & - & - & - & - & - & $\mathbb{9} 300 \mathrm{~A}$ & $39.67 \mathrm{bA}$ \\
\hline- & - & - & - & - & - & - & $\mathrm{I}[300 \mathrm{P}$ & $54.00 \mathrm{bB}$ \\
\hline PS & $0.15^{\mathrm{ns}}$ & $16.31^{*}$ & $7.99^{*}$ & $4.23^{*}$ & $1.87^{\mathrm{ns}}$ & $0.27^{\mathrm{ns}}$ & - & $14.83^{*}$ \\
\hline MF & $1.10^{\mathrm{ns}}$ & $0.38^{\mathrm{ns}}$ & $1.81^{\mathrm{ns}}$ & $0.83^{\mathrm{ns}}$ & $1.10^{\mathrm{ns}}$ & $1.37^{\mathrm{ns}}$ & - & $53.1^{*}$ \\
\hline PS $x$ MF & $0.55^{\mathrm{ns}}$ & $4.55^{\mathrm{ns}}$ & $1.10^{\mathrm{ns}}$ & $0.72^{\mathrm{ns}}$ & $2.41^{\mathrm{ns}}$ & $0.73^{\mathrm{ns}}$ & - & $3.04^{*}$ \\
\hline Block & $0.04^{\mathrm{ns}}$ & $0.69^{\text {ns }}$ & $0.20^{\mathrm{ns}}$ & $0.54^{\mathrm{ns}}$ & $1.25^{\mathrm{ns}}$ & $0.91^{\text {ns }}$ & - & $1.87^{\mathrm{ns}}$ \\
\hline $\mathrm{CV}(\%)$ & 22.56 & 19.80 & 7.05 & 18.11 & 21.09 & 13.48 & - & 14.33 \\
\hline \multicolumn{9}{|c|}{118 DAS } \\
\hline$\$ 0$ & $3.80 \mathrm{a}$ & $0.48 \mathrm{a}$ & $24.67 \mathrm{a}$ & $128 \mathrm{a}$ & $0.11 \mathrm{a}$ & $0.39 a$ & II0 A & $16.00 \mathrm{aA}$ \\
\hline$\$ 100$ & $3.92 \mathrm{a}$ & $1.03 \mathrm{a}$ & $28.50 \mathrm{ab}$ & $149 \mathrm{ab}$ & $0.10 \mathrm{a}$ & $0.34 \mathrm{a}$ & g0 P & $31.33 \mathrm{aB}$ \\
\hline$\S 200$ & $3.92 \mathrm{a}$ & $1.73 \mathrm{ab}$ & $28.50 \mathrm{ab}$ & $159 \mathrm{ab}$ & $0.10 \mathrm{a}$ & $0.40 \mathrm{a}$ & $\Phi 100 \mathrm{~A}$ & $32.67 \mathrm{bA}$ \\
\hline$\S 300$ & $4.20 \mathrm{a}$ & $2.57 \mathrm{~b}$ & $31.50 \mathrm{~b}$ & $181 \mathrm{~b}$ & $0.10 \mathrm{a}$ & $0.30 \mathrm{a}$ & II100 P & $39.67 \mathrm{aA}$ \\
\hline$\S \mathrm{A}$ & $4.23 \mathrm{~A}$ & $1.68 \mathrm{~A}$ & $28.08 \mathrm{~A}$ & $152 \mathrm{~A}$ & $0.09 \mathrm{~A}$ & $0.38 \mathrm{~A}$ & $\mathrm{I} 200 \mathrm{~A}$ & $34.33 \mathrm{bA}$ \\
\hline$\S \mathrm{P}$ & $3.69 \mathrm{~A}$ & $1.82 \mathrm{~A}$ & $28.50 \mathrm{~A}$ & $156 \mathrm{~A}$ & $0.11 \mathrm{~A}$ & $0.34 \mathrm{~A}$ & $\mathrm{I}[200 \mathrm{P}$ & $41.66 \mathrm{aA}$ \\
\hline- & - & - & - & - & - & - & $\mathbb{I}[300 \mathrm{~A}$ & $34.67 \mathrm{bA}$ \\
\hline- & - & - & - & - & - & - & I[300 P & $45.66 \mathrm{aA}$ \\
\hline PS & $0.38^{\mathrm{ns}}$ & $21.74^{*}$ & $7.55^{*}$ & $3.74^{*}$ & $0.02^{\mathrm{ns}}$ & $2.28^{\mathrm{ns}}$ & - & $7.59^{*}$ \\
\hline $\mathrm{MF}$ & $1.30^{\mathrm{ns}}$ & $0.09^{\text {ns }}$ & $0.17^{\mathrm{ns}}$ & $0.16^{\mathrm{ns}}$ & $0.92^{\mathrm{ns}}$ & $2.08^{\mathrm{ns}}$ & - & $14.30^{*}$ \\
\hline PS $x$ MF & $0.61^{\mathrm{ns}}$ & $0.65^{\mathrm{ns}}$ & $0.83^{\mathrm{ns}}$ & $1.81^{\mathrm{ns}}$ & $0.33^{\mathrm{ns}}$ & $1.23^{\mathrm{ns}}$ & - & $2.52 *$ \\
\hline Block & $0.14^{\mathrm{ns}}$ & $0.98^{\mathrm{ns}}$ & $2.74^{\mathrm{ns}}$ & $0.26^{\mathrm{ns}}$ & $2.5^{\mathrm{ns}}$ & $1.97^{\mathrm{ns}}$ & - & $0.71^{\mathrm{ns}}$ \\
\hline $\mathrm{CV}(\%)$ & 16.64 & 18.37 & 8.82 & 18.36 & 17.89 & 10.48 & - & 19.08 \\
\hline
\end{tabular}

$\S$ : means $(\mathrm{n}=6)$ followed by the same lower-case letters in column do not differ by Tukey test at $5 \%$ significance for PS and means $(\mathrm{n}=$ 12) followed by the same upper-case letters in column do not differ by Tukey test at $5 \%$ significance for MF.

II: means $(n=3)$ followed by the same lower-case letters in column do not differ by Tukey test at $5 \%$ significance for interaction sliced PS in MF and the same upper-case letters in column do not differ in Tukey test at 5\% significance for interaction sliced MF in PS

$\dagger$ : Transformation $x^{1 / 2}, \mathrm{~A}$ : absence of MF, P: presence of MF *: Significant at 5\%; ns: not significant at 5\%, CV: coefficient of variation.

According to Raij (1991), the OM contents were classified as high, since they were superior to $25 \mathrm{~g}$. $\mathrm{dm}^{-3}$ and PS doses increased the CEC of the soil throughout this experiment (Table 3). Its application provided an increase in the $\mathrm{OM}$ and hence CEC, which could be associated with the BOD present in the PS (Table 1) (Queiroz et al. $\left.2004\left(\mathrm{BOD}=400 \mathrm{mg} \cdot \mathrm{L}^{-1} \cdot \mathrm{day}^{-1}\right)\right)$.
There was significant interaction between PS $\mathrm{x}$ MF for P at 59 and 118 DAS (Table 3). Despite this, $\mathrm{P}$ concentrations increased with PS doses and MF. Such increase occurs due to the importance to set a range determination of PS doses when $\mathrm{N}$ is used as a nutrient reference (Hountin et al. 2000; Scheffer-Basso et al. 2008; Ceretta et al. 2010 (N $\left.=5,110 \mathrm{mg} \cdot \mathrm{L}^{-1}\right)$, Djodjic et al. 2004; Berwanger et al. 2008). 
If soil samples were taken from the surface layers, $\mathrm{P}$ concentration could be higher, since it could quickly interact with the mineral fraction (Berwanger et al. 2008). The PS and MF provided significant differences in $\mathrm{K}^{+}$concentrations at 59 and 118 DAS (Table 2), according to Queiroz et al. (2004) $\left(\right.$ BOD $=400 \mathrm{mg} \cdot \mathrm{L}^{-1} \cdot$ day $\left.^{-1}\right)$, Assmann et al. (2009) $\left(\mathrm{N}=4,200 \mathrm{mg} . \mathrm{L}^{-1}, 2700 \mathrm{mg} \cdot \mathrm{L}^{-1}, 6,780\right.$ $\mathrm{mg} . \mathrm{L}^{-1}$ and 3,150 mg. $\mathrm{L}^{-1}$ ) and Bertol et al. (2004), $\mathrm{K}^{+}$has low reactivity with soil, hence, it could enhance the mobility. Raij (1991) described that the higher the ions valence, the higher their bias to be fixed in the oil, according to the following order: $\mathrm{Al}^{+3}>\mathrm{Ca}^{+2}>\mathrm{Mg}^{2+}>\mathrm{NH}^{4+}>\mathrm{K}^{+}>\mathrm{H}^{+}>$ $\mathrm{Na}^{+}$.

There were no significant differences for $\mathrm{Cu}^{2+}$ when compared with the PS and MF factors (Table 3). However, at 0,59 and $118 \mathrm{DAS}, \mathrm{Cu}^{2+}$ contents were considered high and superior to $0.8 \mathrm{mg} \mathrm{dm}^{-3}$ (Raij et al. 2001). In general, there was some $\mathrm{Cu}^{2+}$ reduction throughout the experiment according to the amount required by the crop, because for each ton of soybean produced, $26 \mathrm{~g}$ of $\mathrm{Cu}^{2+}$ were exported (Malavolta 2006). Queiroz et al. (2004) $\left(\mathrm{BOD}=400 \mathrm{mg} \cdot \mathrm{L}^{-1} \cdot\right.$ day $\left.^{-1}\right)$ observed that $\mathrm{Cu}^{2+}$, unlike other nutrients, had its concentration decreased due to its absorption by the crop as well as complexation with organic matter from the PS applied on the soil. Such complexation was mainly due to humic and fuvic acids. Another important factor is the high $\mathrm{Cu}^{2+}$ adsorption to organic and inorganic colloids on the soil (Silva and Mendonça 2007). In general, the observed $\mathrm{OM}$ and $\mathrm{Zn}^{2+}$ contents were classified as high $\left(25 \mathrm{~g} . \mathrm{dm}^{-3}\right)$ and low (> 600 mg.dm ${ }^{-3}$ ) (Raij et al. 2001).

There was no significant difference among the treatments regarding concentrations of $\mathrm{Ca}^{2+}, \mathrm{Mg}^{2+}$, $\mathrm{Na}^{+}, \mathrm{EC}$ and SAR. On the other hand, Queiroz et al. (2004) $\left(\mathrm{BOD}=400 \mathrm{mg} \cdot \mathrm{L}^{-1} \cdot \mathrm{day}^{-1}\right)$ observed significant effects of the treatment with the PS since there was higher concentration of $\mathrm{P}, \mathrm{K}^{+}, \mathrm{Na}^{+}$ and $\mathrm{Zn}^{2+}$ in the soil and a decrease of $\mathrm{Mg}^{2+}$ and $\mathrm{Cu}^{2+}$, while $\mathrm{Ca}^{2+}$ concentration was the same. Scherer et al. (2007) $\left(\mathrm{N}=3,810 \mathrm{mg} \cdot \mathrm{L}^{-1}\right)$ reported an increase in $\mathrm{Ca}^{2+}$ and $\mathrm{Mg}^{2+}$ concentrations with PS application. Scherer and Nesi (2009) $(\mathrm{N}=$ 3,100 mg. $\mathrm{L}^{-1}$ ) observed an increase of $\mathrm{P}, \mathrm{K}^{+}, \mathrm{Ca}^{2+}$, $\mathrm{Mg}^{2+}, \mathrm{Zn}^{2+}$ and $\mathrm{Cu}^{2+}$, but these authors sampled the surface layers of the soil.

The $\mathrm{Na}^{+}$did not increase throughout this experiment, although it showed a high concentration in the PS when compared with the soil (Table 2). The studied $\mathrm{Na}^{+}$concentrations were not enough to cause environmental problems (Lamb 2001). In this context, therefore, EC and SAR did not show problems of salinity and sodicity (Richards 1954).

\section{Parameters of leachate}

Minimum and maximum concentrations of leachate material from lysimeters according to the treatments, during the experiment, are shown in Figures 1 and 2. These show the limits in order to evaluate the environmental problems (BRASIL 2005; BRASIL 2008; Basso et al. 2005; Ayres and Westcot 1991). Parameters as $\mathrm{pH}, \mathrm{N}, \mathrm{K}^{+}, \mathrm{Ca}^{+2}$, $\mathrm{Mg}^{+2}, \mathrm{Cu}^{+2}$ and $\mathrm{Zn}^{+2}$ were not significant at $5 \%$ when compared with the treatments. However, $\mathrm{NO}_{3}{ }^{-}, \mathrm{P}, \mathrm{Na}^{+}, \mathrm{EC}$ and SAR showed significant changes when compared with the PS treatments combined with MF (Figures 1 and 2).

Considering the limits used to analyze the environmental problems for the leachate material, $\mathrm{NO}_{3}{ }^{-}, \mathrm{Zn}^{+2}, \mathrm{EC}$ and SAR showed no risk for the groundwater contamination (Figures 1 and 2). However, ions such as $\mathrm{P}$ and $\mathrm{Cu}^{+2}$ presented environmental risks to surface waters (Fig. 1). Other ions such as $\mathrm{NO}_{3}^{-}, \mathrm{P}, \mathrm{Na}^{+}, \mathrm{EC}$ and SAR although did not present extreme contents when compared with the applied environmental limits, increased their concentrations over the time with subsequent PS applications (Figures 1 and 2). Thus, in an intermediate and long term, these parameters could cause environmental problems. $\mathrm{NO}_{3}^{-}$concentration in the leachate (Fig. 1) increased significantly with the PS throughout the experiment (Basso et al. 2005). According to Paul and Zebarth (1997), nitritation and nitratation could occur up from 20 days after PS application and the change in $\mathrm{N}$ was mainly influenced by the temperature and soil moisture.

\section{Agronomic parameters}

There was a high increase in the yield of soybean, although PS did not show a significant effect on it when compared with PS treatment (Table 3).

In soil, $\mathrm{N}$ content from the PS was not a limiting factor in soybean yield, even though it was a leguminous, since the highest PS rate (3.01 ton. $\mathrm{h}^{-1}$ ) showed similar changes to the highest observed mean (3.06 ton.ha ${ }^{-1}$ ). Schmidt et al. (2000; 2001) applied PS doses in several places as well as in several soybean varieties and observed similar results. 

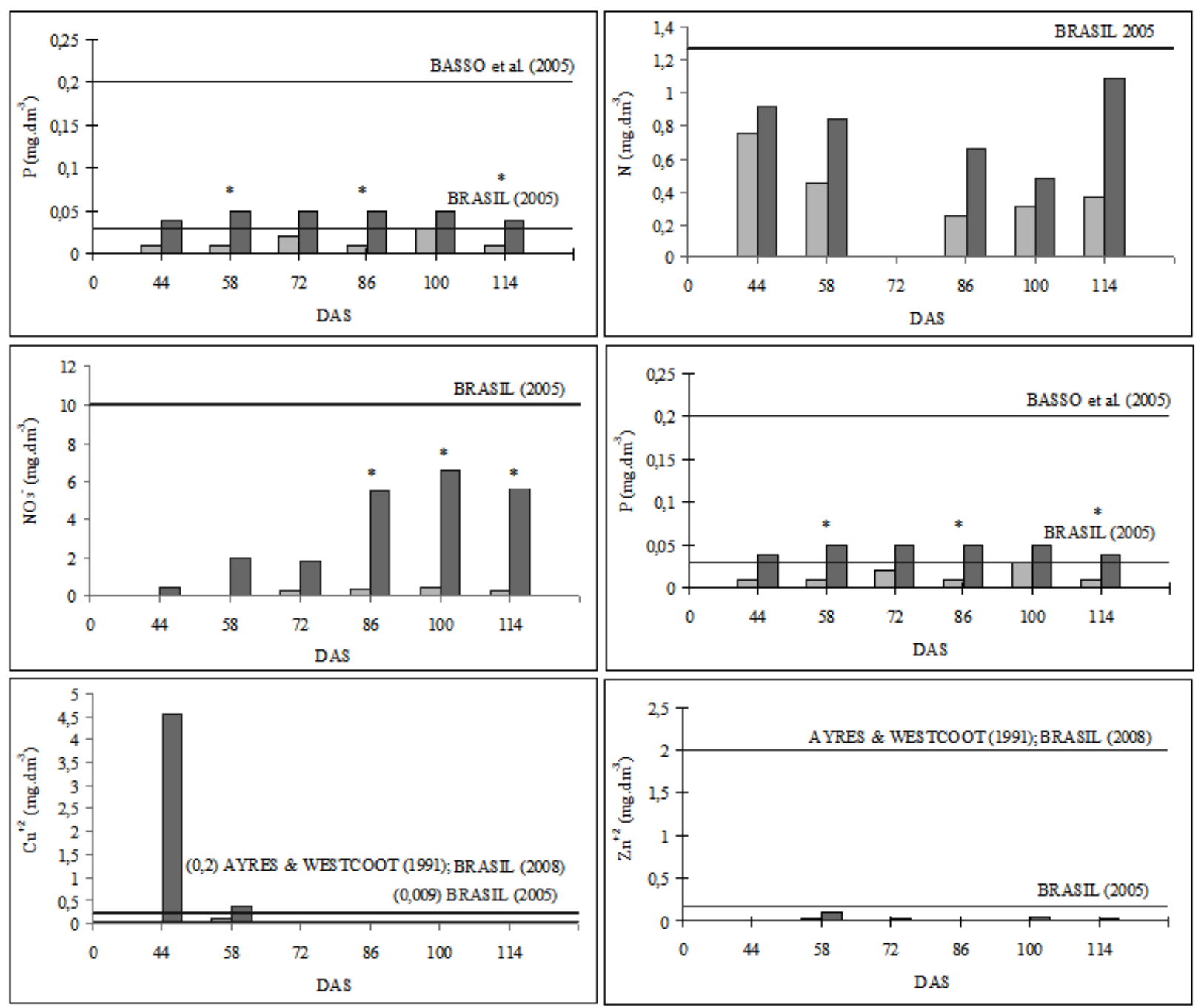

* Significant effect at 5\%, when compared with treatments (PS * MF); DAS - Days after sowing.

Figure 1 - Minimum and maximum contents recorded in parameters as $\mathrm{pH}, \mathrm{N}, \mathrm{NO}_{3}{ }^{-}, \mathrm{P}, \mathrm{Cu}^{+2}, \mathrm{Zn}^{+2}$, compared with treatments, in the leachate material, according to each sampled period.

$\underline{\text { Table } 4 \text { - Analysis of variance (F) and means test for soybean yield. }}$

\begin{tabular}{|c|c|}
\hline & Yield (ton.ha ${ }^{-1}$ ) \\
\hline$\$ 0$ & $2.62 \mathrm{a}$ \\
\hline$\S 100$ & $2.82 \mathrm{a}$ \\
\hline$\$ 200$ & $2.68 \mathrm{a}$ \\
\hline$\$ 300$ & $3.01 \mathrm{a}$ \\
\hline$\S \mathrm{A}$ & $2.51 \mathrm{~A}$ \\
\hline$\S \mathrm{P}$ & $3.06 \mathrm{~B}$ \\
\hline \multicolumn{2}{|l|}{ F Values } \\
\hline PS & $0.90^{\mathrm{ns}}$ \\
\hline MF & $9.24^{*}$ \\
\hline $\mathrm{PS} \times \mathrm{MF}$ & $0.53^{\mathrm{ns}}$ \\
\hline Block & $2.21^{\mathrm{ns}}$ \\
\hline $\mathrm{CV}$ & 15.99 \\
\hline
\end{tabular}

$\S$ : means $(n=6)$ followed by the same lower-case letters in column do not differ by Tukey test at $5 \%$ significance for PS and means $(n=$ 12) followed by the same upper-case letters in column do not differ by Tukey test at $5 \%$ significance for MF. A: absence of MF, P: presence of $\mathrm{MF} *$ : Significant at $5 \%$; ns: not significant at $5 \%, \mathrm{CV}$ : coefficient of variation. 

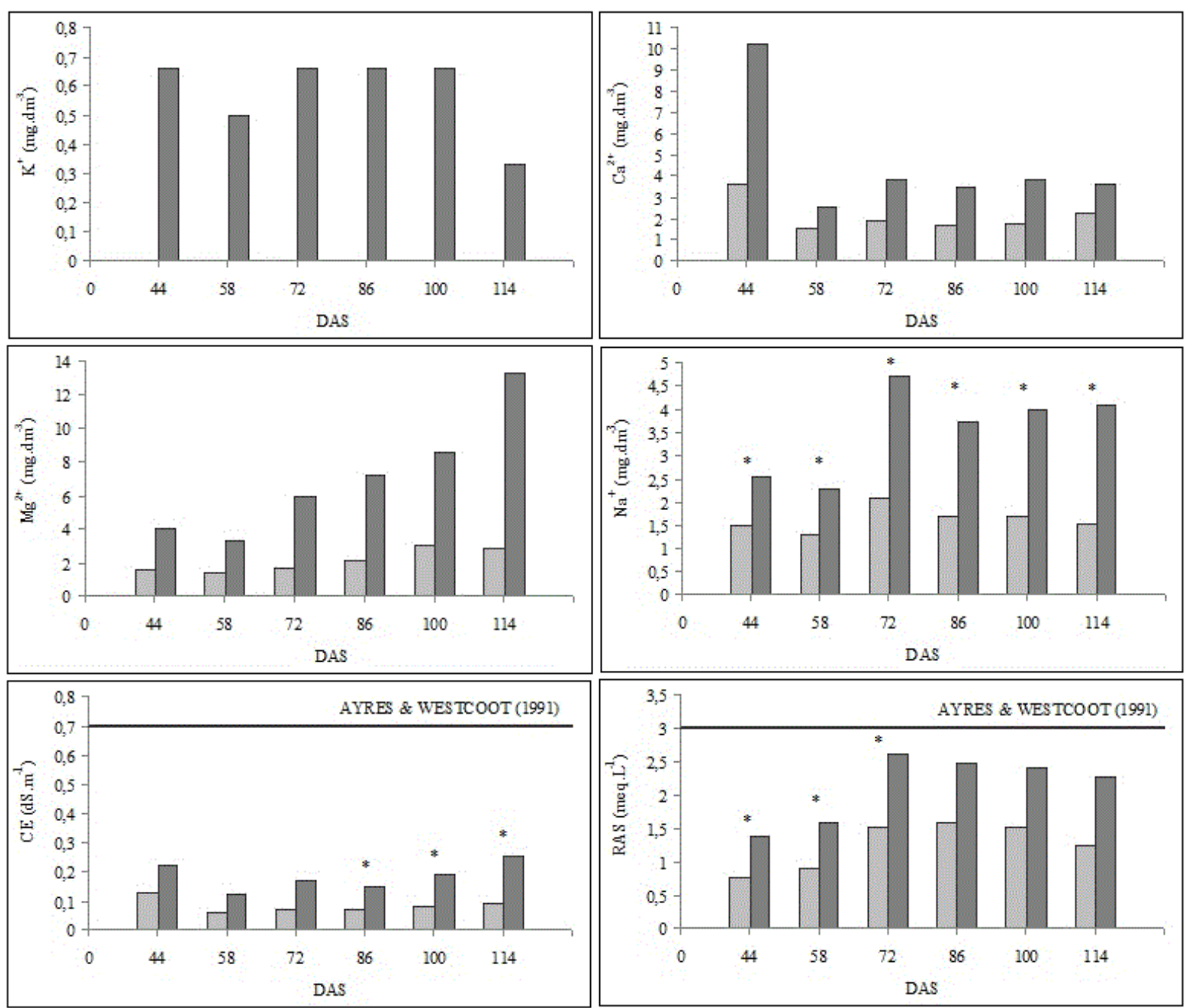

* Significant effect at 5\% of significance; DAS - Days after sowing.

Figure 2 - Minimum and maximum parameters obtained in $\mathrm{K}^{+}, \mathrm{Ca}^{2+}, \mathrm{Mg}^{2+}, \mathrm{Na}^{+}, \mathrm{EC}, \mathrm{SAR}$, compared with treatments, in the leachate material, according to each sampled period.

\section{CONCLUSIONS}

From the results, it could be concluded that In typical Rhodic Hapludox soil, the apploication of pig slurry resulted in increase of $\mathrm{pH}$, nitrate, phosphorus, potassium, zinc, organic matter, and cation exchange capacity, while mineral fertilizer increased phosphorus and potassium in soil.

In leachate, the environmental risks of groundwater contamination were detected; however, for nitrate, phosphorus, sodium, copper, sodium adsorption ratio and electrical conductivity, special attention should be given in an intermediate and long term where PS was applied.
Chemical fertilizer increased significantly the yield of soybean and pig slurry application was a predominant factor in the increase of its yield.

\section{REFERENCES}

Aita C, Giacomini SJ, Hubner AP, Chiapinotto IC, Fries MR. Consorciação de plantas de cobertura antecedendo o milho em plantio direto: I - Dinâmica do nitrogênio no solo. Rev Bras Ciênc Solo. 2004; 28(4): 739-749.

AMERICAN PUBLIC HEALTH ASSOCIATION APHA; AWWA, WEF. Standard methods for the examination of water and wastewater. 20nd ed. Washington: American Public Health Association; (1998). 
Anami M H, Sampaio SC, Suszek M, Gomes SD, Queiroz MMF. Deslocamento miscível de nitrato e fosfato proveniente de água residuária da suinocultura em colunas de solo. Rev Bras Eng Agríc Ambient. 2008; 12(1): 75-80.

Ashworth DJ, Alloway BJ. Complexation of copper by sewage sludge-derived dissolved organic matter: effects on soil sorption behavior and plant uptake. Water Air Soil Pollut. 2007; 182(1): 187-196.

Askegaard M, Olesen JE, Rasmussen IA, Kristensen K. Nitrate leaching from organic arable crop rotations is mostly determined by autumn field management. Agr Ecosyst Environ. 2011; 142(3-4): 149-160.

Assmann JM, Braida JA, Cassol LC, Magiero, E.C.; Manteli C, Griz E. Produção de matéria seca de forragem e acúmulo de nutrientes em pastagem anual de inverno tratada com esterco líquido de suínos. Ciênc Rural. 2009; 39(8): 2408-2416.

Assmann TS, Assmann JM, Cassol LC, Diehl RC, Manteli C, Magiero C. Desempenho da mistura forrageira de aveia-preta mais azevém e atributos químicos do solo em função da aplicação de esterco líquido de suínos. Rev Bras Ciênc Solo. 2007; 31(6): 1515-1523.

Ayers R S, Westcoot D W. A qualidade da água na agricultura. Campina Grande: UFPB; 1991.

Basso CJ, Ceretta CA, Durigon R, Poletto N, Girotto E. Dejeto líquido de suínos: II - perdas de nitrogênio e fósforo por percolação no solo sob plantio direto. Ciênc Rural. 2005; 35(6): 1296-1304.

Bergström L, Kirchmann H. Leaching and crop uptake of nitrogen and phosphorus from pig slurry as affected by different application rates. $J$ Environ Qual. 2006; 35(5): 1803-1811.

Bertol I, Guadagnin JC, Cassol PC, Amaral AJ. Barbosa, F. T. Perdas de fósforo e potássio por erosão hídrica em um inceptisol sob chuva natural. Rev Bras Ciênc Solo. 2004; 28(3): 485-494.

Berwanger AL, Ceretta CA, Santos DR. Alterações no teor de fósforo no solo com aplicação de dejetos líquidos de suínos. Rev Bras Ciênc Solo. 2008; 32(6): 2525-2532.

BRASIL. Ministério do Meio Ambiente. Conselho Nacional do Meio Ambiente. Resolução no 357, de 17 de março de 2005. Dispõe sobre a classificação dos corpos de água e diretrizes ambientais para o seu enquadramento bem como estabelece condições e padrões de lançamento de efluentes e dá outras providências. Diário oficial da União, 18 de março de 2005.

BRASIL. Ministério do Meio Ambiente. Conselho Nacional do Meio Ambiente. Resolução no 396, de 03 de abril de 2008. Dispõe sobre a classificação dos corpos de água e diretrizes ambientais para o seu enquadramento das águas subterrâneas e dá outras providências. Diário oficial da União, 07 de abril de 2008.
Buckey KE, Mohr RM, Therrien MCY. Yield and quality of aot in response to varying rates of swine slurry. Can J Plant Sci. 2010; 90(5): 645-653.

Caires EF, Kusman T, Barth G, Garbuio FJ, Padilha JM. Alterações químicas do solo e resposta do milho à calagem e aplicação de gesso. Rev Bras Ciênc Solo. 2004; 28(1): 125-136.

Caovilla F, Sampaio SC, Smanhotto A, Nóbrega LHP, Queiroz MMF, Gomes BM. Características químicas de solo cultivado com soja e irrigado com água residuária da suinocultura. Rev Bras Eng Agríc Ambient. 2010; 14(7): 692-697.

Caovilla FA, Sampaio SC, Pereira JO, Vilas Boas MA, Gomes BM, Figueiredo AC. Lixiviação de nutrientes proveniente de águas residuárias em colunas de solo cultivado com soja. Re. Bras Eng Agríc Ambient. 2005; 9(Suplem.): 283-287

Ceretta CA, Lorensini F, Brunetto G, Girotto E, Gatiboni LC, Lourenzi CR, Tiecher TL, Conti L, Trentin G, Miotto, A. Frações de fósforo no solo após sucessivas aplicações de dejetos de suínos em plantio direto. Pesqui Agropecu Bras. 2010; 45(6): 593-602.

Dal Bosco TC, Sampaio SC, Opazo MAU, Gomes SD, Nóbrega LHP. Aplicação de água residuária de suinocultura em solo cultivado com soja: cobre e zinco no material escoado e no solo. Eng Agríc. 2008; 28(4): 699-709.

Daudén A, Quílez D. Pig slurry versus mineral fertilization on corn yield and nitrate leaching in a Mediterranean irrigated environment. Eur J Agr. 2004; 21: 7-19.

Deng L, Zheng P, Chen Z, Mahmood Q. Improvement in post-treatment of digested swine wastewater. Bioresour Technol. 2007; 99(8): 3136-3145.

Díez JA, Heraiz P, Muñoz MJ, Torre A, Vallejo A. Impact of pig slurry on soil properties, water salinization, nitrate leaching and crop yield in a fouryear experiment in Central Spain. Soil Use Manage. 2004; 20(4): 444-450.

Djodjic F, Börling K, Bergström L. Phosphorus leaching in relation to soil type and soil phosphorus content. J Environ Qual. 2004; 33: 678-684.

Doblinski AF, Sampaio SC, Silva VR, Nóbrega LH, Gomes SD, Dal Bosco TC. Nonpoint source pollution by swine farming wastewater in bean crop. Rev Bras Eng Agríc Ambient. 2010; 14(1): 87-93.

EMBRAPA. Sistema Brasileiro de Classificação de Solo. 2nd ed. Rio de Janeiro; 2006.

Freitas WS, Oliveira RA, Cecon PR, Pinto FA, Galvão JCC. Efeito da aplicação de águas residuárias de suinocultura em solo cultivado com milho. Eng Agric. 2005; 13(2): 95-102.

Gatiboni LC, Brunetto G, Kaminski J, Rheinheimer DS, Ceretta CA, Basso CJ. Formas de fósforo no solo após sucessivas adições de dejeto líquido de suínos em pastagem natural. Rev Bras Ciênc Solo. 2008; 32(4): 1753-1761. 
Giacomini SJ, Aita C, Jantalia CP, Urquiaga, S. Aproveitamento pelo milho do nitrogênio amoniacal de dejetos líquidos de suínos em plantio direto e preparo reduzido do solo. Pesq Agrop Bras. 2009; 44(7): 761-8.

Gomes, E.R.S.; Sampaio, S.C.; Corrêa, M.M.; Vilas Boas, M.A.; Alves, L.F.A.; Sobrinho, T.S. Movimento de nitrato proveniente de água residuária em colunas de solos. Eng Agríc. 2004; 24(3): 557568.

Gräber I, Hansen JF, Olesen SE, Hans JP, Ostergaard HS, Krogh L. Accumulation of copper and zinc in Danish agricultural soils in intensive pig production areas. Dan J of Geog. 2005; 105(2): 15-22.

Guarçoni, A.; Mendonça, E.S. Capacidade tampão de pH do solo e disponibilidade de fósforo pela adição de composto orgânico. Magistra. 2003; 15(2).

Hao X, Zhou D, Sun L, Li L, Zhang H. Dynamic chemical characteristics of soil solution after pig manure application: A column study. J Envir Sci H. 2008; 43(5): 429-436.

Heathwaite L, Sharpley A, Gburek WA. A conceptual approach for integrating phosphorus and nitrogen management at watershed scales. J Environ Qual. 2000; 23(2): 337-343.

Hountin, J.A.; Karam, A.; Couillard, D.; Cescas, M.P. Use of a fractionation procedure to assess the potential for $\mathrm{P}$ movement in a soil profile after 14 years of liquid pig manure fertilization. Agric and Ecos Environ. 2000; 78(1): 77-84.

Hsu JH, Lo SL. Effect of composting on characterization and leaching of copper, manganese and zinc form swine manure. Environ Pollution. 2000; 114(1): 119-127.

Hue NV, Licudine DL. Amelioration of subsoil acidity through surface application of organic manures. $J$ Environ Qual. 1999; 28(2): 623-632.

IAPAR. Cartas Climáticas do Estado do Paraná. Londrina: IAPAR; 2000.

Jondreville C, Revy PS, Dourmad JY. Dietary means to better control the environmental impact of copper and zinc by pigs from weaning to slaughter. Livestock Produc Sci. 2003; 84(2): 147-156.

Longo AJ, Sampaio SC, Suszek M. Equação de chuvas intensas e precipitação provável para o município de Cascavel PR. Varia Scientia. 2006; 6(12): 16-30.

Maggi CF, Freitas CLF, Sampaio SC, Dieter J. Lixiviação de nutrientes em solo cultivado com aplicação de água residuária de suinocultura. Rev Bras Eng Agríc Ambient. 2011; 15(2): 170-177.

Malavolta E. Manual de nutrição mineral de plantas. São Paulo: Agronômica Ceres; 2006.

Mattias JL, Ceretta CA, Nesi CN, Girotto E, Trentin EE, Lourenzi CR, Vieira RCB. Copper, zinc and manganese in soils of two watersheds in Santa Catarina with intensive use of pig slurry. Rev Bras Ciênc Solo. 2010; 34(4): 1445-1454.
Medeiros S, Soares AA, Ferreira PA, Neves JCL, Matos AT, Souza JAA. Utilização de água residuária de origem doméstica na agricultura: estudo das alterações químicas do solo. Rev Bras Eng Agríc Ambient. 2005; 9(4): 603-612.

Mori HF, Favaretto N, Pauletti V, Dieckow J, Santos WL. Perda de água, solo e fósforo com aplicação de dejeto líquido bovino em latossolo sob plantio direto e com chuva simulada. Rev Bras Ciênc Solo. 2009; 33(1): 189-198.

Müller K, Magesan GN, Bolan NS. A critical review of the influence of effluent irrigation on the fate of pesticides in soil. Agric Ecosyst Environ. 2007; 120(2-4), 93-116.

Oliveira RA, Campelo PLG, Matos AT, Martinez MA, Cecon PR. Influência da aplicação de águas residuárias de suinocultura na capacidade de infiltração de um solo podzólico vermelho-amarelo. Rev Bras Eng Agríc Ambient. 2000; 58(4): 263-267.

Paul JW, Zebarth BJ. Denitrification and nitrate leaching during the fall and winter following dairy cattle slurry application. Canad J Soil Sci. 1997; 77(2): 231-240.

Primavesi O, Primavesi AC, Corrêa LA, Silva AG, Cantarella H. Lixiviação de nitrato em pastagem de coastcross adubada com nitrogênio. Rev Bras Zoot. 2006; 35(3): 683-690.

Prior M, Smanhotto A, Sampaio SC, Nóbrega LHP, Opazo MAU, Dieter J. Acúmulo e percolação de fósforo no solo devido à aplicação de água residuária da suinocultura na cultura do milho (Zea mays L.). Pesq Aplic Agrotec. 2009; 2(1): 89-96.

Queiroz FM, Matos AT, Pereira OG, Oliveira RA. Características químicas de solo submetido ao tratamento com esterco líquido de suínos e cultivado com gramíneas forrageiras. Ciênc Rural. 2004; 34(5): 1487-1492.

Raij B V. Fertilidade do solo e adubação. Piracicaba: Ceres; 1991.

Raij BV, Andrade JC de, Cantarella H, Quaggio JA. Análise química para avaliação da fertilidade de solos tropicais. Campinas: Instituto Agronômico - IAC; 2001.

Ribeiro AG, Galbiatti JA. Contaminação por nitrato e sódio da água percolada e da planta de alface irrigada com água residuária. Holos Environ. 2004; 4(1): 5667.

Richards LA. Diagnosis and improvement of saline and alkali soils. Washington: United States Salinity Laboratory Staff, (United States Department of Agriculture Handbook, 60); 1954.

Rossi P, Miranda JH, Duarte SN. Curvas de distribuição de efluentes do íon nitrato em amostras de solo deformadas e indeformadas. Eng Agríc. 2007; 27(3): 675-682.

Sampaio SC, Caovilla FA, Opazo MAU, Nóbrega LHP, Suszek M, Smanhotto A. Lixiviação de íons em 
colunas de solo deformado e indeformado. Eng Agríc. 2010b; 30(1): 150-159.

Sampaio SC, Fiori MGS, Opazo MAU, Nóbrega LHP. Comportamento das formas de nitrogênio em solo cultivado com milho irrigado com água residuária da suinocultura. Eng Agríc. 2010a; 30(1): 138-149.

Scheffer-Basso SM, Scherer CV, Ellwanger MF. Resposta de pastagens perenes à adubação com chorume suíno: pastagem natural. Rev Bras de Zoot. 2008; 37(2): 221-227.

Scherer EE, Aita C, Baldissera IT. Avaliação da qualidade do esterco líquido de suínos da região oeste catarinense para fins de utilização como fertilizante. Epagri; 1996.

Scherer EE, Baldissera IT, Nesi CN. Propriedades químicas de um latossolo vermelho sob plantio direto e adubação com esterco de suínos. Rev Bras Ciênc Solo. 2007; 31(1): 123-131.

Scherer EE, Nesi NC. Características químicas de um latossolo sob diferentes sistemas de preparo e adubação orgânica. Bragantia. 2009; 68(3): 715-721.

Schmidt JP, Lamb JA, Schmitt MA, Randall GW, Orf JH, Gollany HT. Swine Manure Application to Nodulating and Nonnodulatin Soybean. Agron J. 2000; 92(5): 987-992.

Schmidt JP, Lamb JA, Schmitt MA, Randall GW, Orf JH, Gollany HT. Soybean Varietal Response to
Liquid Swine Manure Application. Agron J. 2001; 93(2): 358-363.

Silva IR, Sá Mendonça E. Matéria orgânica do solo. In: Novais RF, Alvarez VVH, Barros NF, Fontes RLF, Cantarutti RB, Neves JCL. eds. Fertilidade do Solo. Viçosa: SBCS; 2007. p. 275-374.

Smanhotto A, Souza AP, Sampaio SC, Nóbrega LHP, Prior P. Cobre e zinco no material lixiviado e no solo com a aplicação de água residuária de suinocultura em solo cultivado com soja. Eng Agríc. 2010; 30(2): 347-357.

Vieira RF, Tanaka RT, Tsai SM, Pérez DV, Silva CMMS. Disponibilidade de nutrientes no solo, qualidade de grãos e produtividade da soja em solo adubado com lodo de esgoto. Pesq Agrop Bras. 2005; 40(9): 919-926.

Wohlengerg EV, Reichert JM, Reinert DJ, Blume E. Dinâmica de agregação de um solo franco-arenoso em cinco sistemas de culturas em rotação e em sucessão. Rev Bras Ciênc Solo. 2004; 28(5): 891-900.

Received: July 09, 2012; Accepted: July 14, 2013. 